NHFO were those deemed not to be appropriate for escalation to ITU.

Conclusion Our experience demonstrates that the mortality of oncology patients on HFNO is high. Although starting HFNO in these patient groups might be appropriate if we can demonstrate evidence of reversible pathology, care needs to be taken in managing the expectations of patients and their families, and where relevant, appropriate palliative input sought.

\section{BUSTING SOME MYTHS OF ONLINE DELIVERY OF PALLIATIVE CARE EDUCATION}

Sian Gallard, Fiona Rawlinson, Mark Stacey*, Dylan Harris, Jo Griffiths, Jo Hayes, James Grose, Jo Richards. Cardiff University, *Health Education and Innovation UK

\subsection{6/spcare-2021-PCC.72}

Background/Introduction There is an urgent need to continue palliative care professional development (CPD) for all those delivering health care, especially in view of health needs of the population currently affected by the Covid-19 pandemic. Face to face teaching, whilst preferable to date, has not been possible. The face to face interaction at the start of a Masters course in Palliative medicine was transitioned to virtual learning. We explored the impact of using technology on the learning experience of participants both in terms of comfort with the virtual environment and its impact on their learning

Methods Prospective evaluation of a redesigned face to face teaching programme. Participants were asked if the virtual learning environment or technology had impacted on their learning using a 5 point scale for each of the sessions. A further delayed evaluation is underway, to explore the continued impact of the sessions.

Results 13 sessions forming a 3 day programme were evaluated. 252 responses were received (45\% response rate) 223/ 252 responded that all was well, 18/252 mentioned some technical difficulties but with no negative impact on learning, 8 mentioned technical issues which did impact on learning, 2 mentioned being uncomfortable with the virtual learning environment with no impact on learning and 1 person described being uncomfortable with negative impact.Results to date of the delayed evaluation have been received from 16 participants (response rate of 32\%).

Conclusions Enabling virtual CPD to continue to skill and enable the health care workforce to deliver palliative care to patients is paramount, especially while Covid-19 restrictions to face to face gatherings continue. With attention to teaching techniques (delivery style, focused content and use of interaction), neither the technology or a virtual learning environment, negatively impact learning for the majority of students. Further exploration of prospective data is underway.

\section{THE USE OF BREAKOUT ROOMS AND INTERACTION TO CREATE A COMMUNITY OF PRACTICE IN A VIRTUAL LEARNING ENVIRONMENT}

Sian Gallard, Fiona Rawlinson, Jo Griffiths, Dylan Harris, Jo Hayes, James Grose, Jo Richards. Cardiff University

10.1136/spcare-2021-PCC.73
Background/Introduction Interaction and a community of practice are integral to postgraduate education. Previously, face to face $(\mathrm{F} 2 \mathrm{~F})$ teaching allowed an opportunity for these factors to evolve naturally. Due to Covid -19 restrictions, this interaction needed to transition to virtual learning for a Palliative Medicine MSc. We explored the use of breakout room and use of a 'chat' function on a virtual learning platform to stimulate interaction and create a community of practice. These features were used within sessions for case based discussions, team building activities and immediate whole group responses to particular palliative care issues and challenges, enabling the cohort of students to build on each other's comments.

Methods Participants were invited to send immediate free-text evaluation of the sessions. These results were screened for comment on breakout rooms, and thematic analysis used to explore feelings towards this teaching strategy.

Results 252 responses were received across all sessions (45\% response rate). Results were screened for reference to breakout rooms and use of the chat function. Thematic analysis revealed that these were positively received ('More breakout rooms', 'really good'), and gave rise to an opportunity for people to interact within a virtual learning environment. Additional unsolicited feedback to the course team indicated satisfaction with the delivery style " I felt like there was still a really nice feeling of camaraderie, and that I got to know a lot of the others with the breakout rooms. I also felt much less inhibited using the chat function than I would putting my hand up in a lecture'

Conclusions Breakout rooms, and the use of the 'chat' function, were found to be an excellent medium with which to facilitate interaction and develop a community of practice. Using all aspects of technology to successfully support delivery of palliative care education can enhance palliative care knowledge during this time when F2F education is not possible.

\section{LEARNING FROM DEATHS: THE STRUCTURED JUDGMENT REVIEW}

${ }^{1}$ Stephanie Lister-Flynn, ${ }^{2}$ Emily Sills. ${ }^{1}$ St Catherine's Hospice, Crawley; ${ }^{2}$ Princess Alice Hospice, Esher

\subsection{6/spcare-2021-PCC.74}

Background Most acute trusts have systems in place to review hospital deaths to identify areas that could be improved and areas of good practice. Following on from the National Mortality Case Record Review, the Learning From Deaths Guidance published in 2017 sets key requirements to ensure organisations effectively respond to and learn from deaths. This includes introduction of structured case record reviews when reviewing deaths. Following this guidance, we set up a monthly educational programme at Woking and Sam Beare Hospice from November 2019-September 2020, including monthly adapted 'Structured Judgment Reviews' (SJR) of nominated deaths.

Methods Each month a patient death was selected from a list that teams felt further discussion would be beneficial. An 'independent reviewer', not directly involved in the care, would objectively review the notes. The phases of care focused on were:

- First assessment

- Ongoing care 